\title{
Examining The Relationship Between Smoking Cigarettes and Experiences of Sleep Disturbances
}

\section{Abstract}

Research has shown there is a relationship between adolescent and young adult cigarette smokers and experiences of sleep disturbances. Participants were $(\mathrm{N}=25,049)$ adolescents and early adulthood participants (Mean age $=15.83, S D=4.53$ ) who answered the questions for the variables in this study. Approximately $55 \%$ of the participants reported significant problems with sleep trouble, such as bad dreams, sleeping restlessly or falling asleep during the day in the past month (29.7\%), two to twelve months (13.8\%), and over a year ago (11.3\%) (measured using the Audio Computer-Assisted SelfInterviews [ACASI]). Approximately $54 \%$ of the same participants reported smoking daily (39.6\%) or some days (14.1\%) (measured using ACASI). The specific methods used in this study was a correlational test of the variables predicted to be positively related. It was hypothesized that there is a positive link between one's cigarette smoking and having significant difficulty with sleeping, such as bad dreams, sleeping restlessly or falling asleep during the day. Consistent with the hypothesis, current cigarette smoking was statistically significantly related to having significant difficulty with sleeping, such as bad dreams, sleeping restlessly or falling asleep during the day, resulting with $r=.073(p<.01)$ at the 0.01 level (1-tailed). The $\mathrm{R}^{2}$ and adjusted $\mathrm{R}^{2}=.005$, and the ANOVA table revealed there is a statistically significant linear relationship between current smoking of cigarettes and having sleep disturbances such as bad dreams, sleeping restlessly or falling asleep during the day. These results suggest that as one's cigarette smoking increases their experience with sleep disturbance, such as bad dreams, sleeping restlessly or falling asleep during the day increases. These results support the rejection of the null hypothesis of there being no statistically significant relationship between current cigarette smoking and having sleep disturbances. There is a weak positive correlation between current cigarette smoking and having sleep disturbances. It is concluded that cigarette smoking among young adults is associated with experiences of sleep disturbances.

\section{Purpose Statement}

The purpose of this study was to determine the correlation between cigarette smoking and sleep disturbances, such as bad dreams, sleeping restlessly or falling asleep during the day.

\section{Introduction}

Research has shown there is a positive relationship between smoking cigarettes and experiences of sleep disturbancesRecent studies that have examined the relationship between smoking cigarettes and sleep disturbances in adolescents.

\section{Literature Review}

\section{Phillips et al. (1995)}

- The study examines the relationship between cigarette smoking, poor sleep habits, and sleep complaints. Several lines of evidence suggest a relationship between cigarette smoking and sleep disturbance. There were 484 people who participated from a random sample of 1000 completed a mail survey. The effects of age and smoking status on sleep, health and daytime function were assessed by multivariate analysis of variance. In conclusion, cigarette smokers were significantly more likely than nonsmokers to report problems such as: going and staying asleep, daytime sleepiness, minor accidents, depression, and high daily caffeine intake.

Costa et al. (2018)

- This review aims to provide an overview of previous research on cigarette smoking and it's effect on sleep to use it as evidence of its affect on the body. Data from 33 articles were organized according to the different ways in which cigarette smoking can affect sleep and allowed us to establish a correlation between sleep disturbances. Further investigations targeting a better understanding of how nicotine could affect neural basis of behavior and biological rhythms are needed

Bellatorre et al. (2017)

- Based on a lack evidence the relationship between sleeping and smoking during adolescence remains unclear and complex. The authors used two-group structural equation modeling to evaluate the longitudinal effects of sleep problems, duration and smoking for White and Black youth. Further research is needed to unravel the complex relationship between the direct effects of nicotine, lifestyle choices that may link smoking, sleep problems, and racial differences.

\section{Hypothesis}

Research Question: Is there a relationship between smoking cigarettes and sleep disturbances?

Hypotheses:

HO: There is no statistically significant relationship between

smoking cigarettes and sleep disturbances

$\mathrm{H} 1$ : There is a statistically significant relationship between smoking cigarettes and sleep disturbances

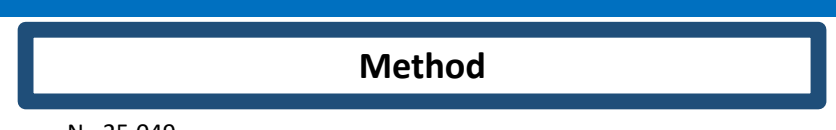

$N=25,049$

Mean Age $=15.83$

Measure:

- Audio Computer-Assisted Self-Interviews (ACASI)
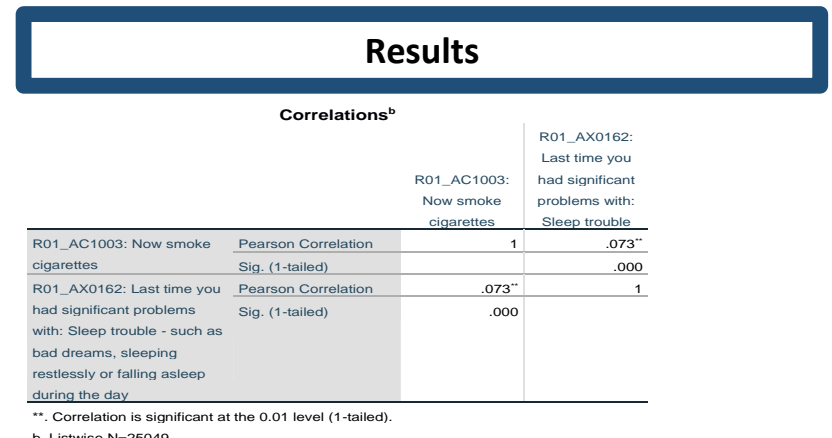

\section{.}

\section{Conclusion}

These results suggest that as one's cigarette smoking increases their experience with sleep disturbance, such as bad dreams, sleeping restlessly or falling asleep during the day increases.

- These results support the rejection of the null hypothesis of there being no statistically significant relationship between current cigarette smoking and having sleep disturbances.

- There is a weak positive correlation between current cigarette smoking and having sleep disturbances.

- It is concluded that cigarette smoking among young adults is associated with experiences of sleep disturbances.

- $\quad$ Future research is needed to determine the complex relationship between smoking and sleep disturbances; there are several other factors that should be considered, such as genetic factors, race/ethnicity, lifestyle choices and SES.

\section{Acknowledgements}

The authors would like to acknowledge our research mentors Professors Victoria $C$ Taylor and Deirdre Thompson, the Humanities, English, and Social Sciences (HESS) Division Chair, Korey Brown, the Psychology Department Coordinator, Professor Sonia Bell, the HESS Division Dean, Dr. Nicole Currier, the Prince George's Community College Humanities, English, and Social Sciences (HESS) Collegian Center, and PGCC faculty and staff in the psychology department. We would also like to acknowledge the use of a secondary data set from the Public Assessment and Tobacco Heath (PATH) Study. The continued support and push enables us to go farther than we could ever dream. Thank you. 